\title{
Targeting NRF2-Governed Glutathione Synthesis for SDHB-Mutated Pheochromocytoma and Paraganglioma
}

\author{
Yang Liu ${ }^{1,+}$, Ying Pang ${ }^{2,+}$, Veronika Caisova ${ }^{2}$, Jianyi Ding ${ }^{1}$, Di Yu ${ }^{1} \mathbb{C}$, Yiqiang Zhou ${ }^{1}$, \\ Thanh-Truc Huynh ${ }^{2}$, Hans Ghayee ${ }^{3,4}$, Karel Pacak ${ }^{2, *, \dagger}$ and Chunzhang Yang ${ }^{1, *,+}$ (D) \\ 1 Neuro-Oncology Branch Center for Cancer Research, National Cancer Institute, Bethesda, MD 20892, USA; \\ yang.liu5@nih.gov (Y.L.); jianyi.ding@nih.gov (J.D.); yudi_1808@dicp.ac.cn (D.Y.); \\ zhou.yiqiang@outlook.com (Y.Z.) \\ 2 Section on Medical Neuroendocrinology, Eunice Kennedy Shriver National Institute of Child Health and \\ Human Development, National Institutes of Health, Bethesda, MD 20892, USA; ying.pang@nih.gov (Y.P.); \\ caisovav@gmail.com (V.C.); huynht@mail.nih.gov (T.-T.H.) \\ 3 Division of Endocrinology, Department of Medicine, University of Florida, Gainesville, FL 32610, USA; \\ hans.ghayee@medicine.ufl.edu \\ 4 Malcom Randall VA Medical Center, Gainesville, FL 32608, USA \\ * Correspondence: karel@mail.nih.gov (K.P.); yangc2@nih.gov (C.Y.); Tel.: +1-301-402-4594 (K.P.); \\ +1-240-760-7083 (C.Y.); Fax: +1-301-402-0884 (K.P.) \\ + These authors contributed equally to this work.
}

Received: 14 January 2020; Accepted: 21 January 2020; Published: 23 January 2020

\begin{abstract}
Succinate dehydrogenase subunit B (SDHB) deficiency frequently occurs in cluster I pheochromocytomas and paragangliomas (PCPGs). SDHB-mutated PCPGs are characterized by alterations in the electron transport chain, metabolic reprogramming of the tricarboxylic cycle, and elevated levels of reactive oxygen species (ROS). We discovered that SDHB-deficient PCPG cells exhibit increased oxidative stress burden, which leads to elevated demands for glutathione metabolism. Mechanistically, nuclear factor erythroid 2-related factor 2 (NRF2)-guided glutathione de novo synthesis plays a key role in supporting cellular survival and the proliferation of SDHB-knockdown $\left(S D H B^{K D}\right)$ cells. NRF2 blockade not only disrupted ROS homeostasis in SDHB-deficient cells but also caused severe cytotoxicity by the accumulation of DNA oxidative damage. Brusatol, a potent NRF2 inhibitor, showed a promising effect in suppressing $S D H B^{K D}$ metastatic lesions in vivo, with prolonged overall survival in mice bearing PCPG allografts. Our findings highlight a novel therapeutic strategy of targeting the NRF2-driven glutathione metabolic pathway against SDHB-mutated PCPG.
\end{abstract}

Keywords: NRF2; glutathione metabolism; SDHB mutation; pheochromocytoma; paraganglioma

\section{Introduction}

Pheochromocytomas and paragangliomas (PCPGs) are neuroendocrine tumors derived from chromaffin cells, which are commonly located in adrenal and extra-adrenal compartments. Genetically, PCPG tumorigenesis is related to genetic alterations in 21 genes, which cluster into three major molecular subtypes on the basis of their signature transcriptomic profiles [1-3]. Cluster I PCPGs are characterized by the activation of the pseudohypoxia-related signaling pathway, which includes mutations in hypoxia-inducible factor 2A (HIF2A), succinate dehydrogenase subunits (SDHA, SDHB, $S D H C, S D H D)$, succinate dehydrogenase complex assembly factor 2 (SDHAF2), von Hippel-Lindau tumor suppressor (VHL), egl-9 prolyl hydroxylases 1 and 2 (EGLN1/2), fumarate hydratase $(F H)$, malate dehydrogenase 2 (MDH2), and the ATP-dependent helicase (ATRX). Meanwhile, cluster II PCPGs 
result from mutations related to the kinase signaling pathway, which includes the RET proto-oncogene, neurofibromin 1 (NF1), Harvey rat sarcoma proto-oncogenes (H-RAS), transmembrane protein 127 (TMEM127), and Myc-associated factor X (MAX) [2,4-10]. Cluster III PCPGs are a recently identified disease subtype, with a transcriptomic signature of Wnt $/ \beta$-catenin signaling [8]. Among all molecular subtypes of PCPGs, genetic abnormalities in SDHx underlie the most aggressive phenotype, with a strong tendency to metastatic disease, tumor multiplicity, and recurrence [11-14].

The current standards of care for metastatic PCPGs usually provide marginal benefit to tumor suppression or elimination and disease outcome including survival. In terms of chemotherapy, a combination regimen including cyclophosphamide, vincristine, and dacarbazine (CVD) is currently recommended as the first-line therapy to manage rapidly progressing metastatic PCPGs, whereas the responses are usually transient [15]. Several longitudinal studies have revealed that although the CVD regimen provides objective tumor responses, it fails to yield improvements in overall survival $[16,17]$. On the other hand, some recent studies have shown that the distinctive transcriptional profile in cluster I PCPG may give rise to a distinctive spectrum of therapeutic options. For example, Hadoux et al. discovered that temozolomide was more effective against metastatic SDHB PCPG due to low $\mathrm{O}^{6}$-alkylguanine DNA alkyltransferase (MGMT) methylation status [18]. In addition, Sulkowski, as well as our group, discovered that SDHB PCPGs exhibit higher sensitivity to a combination regimen involving a poly (adenosine diphosphate-ribose) polymerase (PARP) inhibitor and genotoxic agent, as this type of malignancy exhibits a deficiency of homologous recombination DNA repair and nicotinamide adenine dinucleotide (NAD) metabolism $[19,20]$. Overall, these findings imply that therapeutic regimens can be optimized by targeting the unique molecular signature(s) of cluster I PCPGs.

Nuclear factor (erythroid-derived 2)-like 2 (NFE2L2; NRF2) is a transcriptional factor that governs cellular redox homeostasis by mediating the trans-activation of antioxidant-related genes. Oxidative stress has been recognized as a hallmark of cancers, which promotes tumor growth and malignant progression, especially for cancers with intrinsic altered metabolic signatures, such as SDHB-mutated PCPGs and isocitrate dehydrogenase 1 (IDH1)-mutated malignancies. These cancers tend to develop a dependency on reactive oxygen species (ROS) detoxification pathways to maintain a reasonable ROS level [21]. The distinctive role of the antioxidant pathway in these malignancies suggests that targeting ROS-scavenging pathways could be a valuable anticancer strategy. Owing to the close relevance to therapeutic resistance and detoxification pathways, targeting NRF2 has long been proposed as a potential cancer therapeutic approach $[22,23]$. Meanwhile, pioneering work by Ren et al. suggested that brusatol, a plant-derived natural quassinoid, serves as a potent inhibitor of the NRF2 antioxidant pathway [24]. Considering the dependency of $S D H B$-knockdown $\left(S D H B^{K D}\right)$ PCPG cells on antioxidant scavenging, in the present study, we aimed to determine the effectiveness of brusatol against $S D H B^{K D}$ PCPG.

\section{Results}

\subsection{SDHB Deficiency Altered the Redox Balance in PCPG Cells}

Cancer-associated SDHB mutations have been found to result in the functional disruption of mitochondrial complex II, which causes catastrophic changes to cellular metabolism and redox homeostasis [25-28]. To better understand alterations in the redox status within SDHB-mutated PCPGs, we established $S D H B^{K D}$ PCPG cell lines based on the mouse pheochromocytoma cell line MPC $\left(\mathrm{MPC} S D H B^{K D}\right.$ ) and the human cell line hpheo1 (hpheo1 $S D H B^{K D}$ ). The knockdown efficiency was confirmed by western blot assay (Figure 1A). Further, the loss of $S D H B$ led to substantial compromised oxidative metabolism and accumulation of succinate (Figure S1). Genetic disruption of SDHB resulted in robust accumulation of ROS in mitochondria and cytoplasm. MitoSOX Red staining showed a significant increase in ROS generation in $S D H B^{K D}$ compared to $S D H B$ wild type $\left(S D H B^{W T}\right)$ cells (Figure 1B,C). A similar trend was observed through the direct quantification of intracellular $\mathrm{H}_{2} \mathrm{O}_{2}$, whereas the addition of the exogenous ROS scavengers $\mathrm{N}$-acetylcysteine (NAC) or catalase reduced ROS 
accumulation (Figure 1D). The ROS elevation was relieved by the re-expression of SDHB (Figure S2A,B). To better understand the molecular basis of the redox balance in SDHB-deficient cells, we specifically quantified intracellular glutathione, the major source of ROS detoxification. The glutathione/glutathione disulfide (GSH/GSSG) ratio decreased by over $50 \%$ upon genetic silencing of SDHB, indicating that $S D H B^{K D}$ cells consume GSH for ROS scavenging and convert to more GSSG (Figure 1E and Figure S1C). Consistent with higher demands for glutathione, the expression levels of key enzymes, transcriptional factors, and transporters in the glutathione synthesis pathway, such as NFE2L2, glutamate-cysteine ligase regulatory subunit (GCLM), and cystine/glutamate transporter (SLC7A11), were upregulated in patients with cluster I PCPG (Figure 1F,G). Real-time PCR confirmed that the messenger RNA (mRNA) level of SLC7A11 was increased in cluster I PCPGs (Figure 1H).

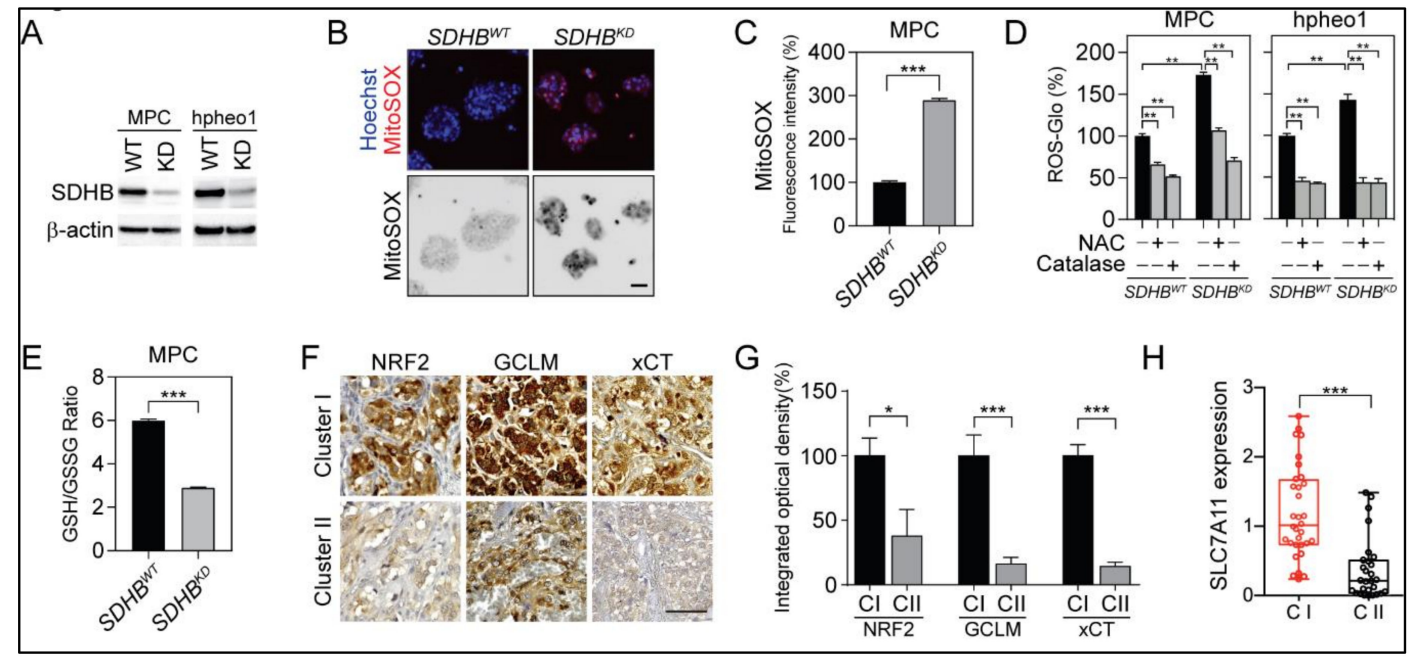

Figure 1. Succinate dehydrogenase subunit B $(S D H B)$ deficiency reprogrammed reactive oxygen species (ROS) homeostasis and glutathione metabolism. (A) Immunoblotting showed the expression levels of $S D H B$ in mouse pheochromocytoma (MPC) and hpheo1 cells. $\beta$-actin was used as internal control. (B) MitoSOX-Red staining showed ROS accumulation in SDHB knock down (SDHB ${ }^{K D}$ ) MPC cells. Bar $=10 \mu \mathrm{m}$. (C) Flow cytometry analysis showed elevated MitoSOX-Red staining in $S D H B^{K D}$ compared to $S D H B$ wild type $\left(S D H B^{W T}\right)$ MPC cells. ${ }^{* *} p<0.001$. (D) ROS quantification showed increased ROS in $S D H B^{K D}$ MPC and hpheo1 cells. Exogenous ROS scavenger $N$-acetylcysteine (NAC) and catalase reduced ROS level. ROS signal was measured and normalized to protein quantification. $* * p<0.01$. (E) Glutathione quantification showed reduction of glutathione/glutathione disulfide (GSH/GSSG) ratio in $S D H B^{K D}$ compared to $S D H B^{W T}$ MPC cells. ${ }^{* * *} p<0.001$. (F) Immunohistochemistry staining showed that nuclear factor erythroid 2-related factor 2 (NRF2), glutamate-cysteine ligase regulatory subunit (GCLM), and cystine/glutamate transporter (SLC7A11, xCT) were increased in cluster I pheochromocytomas and paragangliomas (PCPGs). Bar $=50 \mu \mathrm{m}$. (G) Integrated optical density quantification for results shown in Figure $1 \mathrm{~F}$. For cluster I (CI), $n=4$; for cluster II (CII), $n=4$. ${ }^{*} p<0.05 ;{ }^{* * *} p<0.001$. (H) Quantitative real-time PCR showed that SLC7A11 messenger RNA (mRNA) was increased in cluster I $(\mathrm{C} 1 ; n=8)$ compared to cluster II (CII; $n=7)$ PCPG specimen. ${ }^{* * *} p<0.001$.

\subsection{NRF2 Supported Glutathione De Novo Synthesis in SDHB KD Cells}

The transcription of glutathione synthesis enzymes was regulated by NRF2, and increased levels of glutathione synthesis enzymes suggested functional alterations in NRF2 protein biology and transcriptional activity. To further understand the role of NRF2 in an SDHB-deficient genetic background, we analyzed the activation of NRF2 using an antioxidative response element (ARE) luciferase reporter assay. We found that $S D H B^{K D}$ cells exhibited significantly higher ARE transcriptional activity than $S D H B^{W T}$ cells, suggesting that the transcriptional activity of NRF2 was increased due to $S D H B$ deficiency (Figure 2A). Introduction of $S D H B$ expression decreased ARE luciferase activity in $S D H B^{K D}$ cells (Figure S2C). Quantitative real-time PCR and immunoblotting confirmed that NRF2 
and its downstream target genes, such as GCLM, and SLC7A11 (xCT), were significantly upregulated (Figure 2B,C). In addition, we found that the half-life of NRF2 was prolonged in $S D H B^{K D}$ cells compared to their wild-type counterparts, indicating a more sustained NRF2 activation (Figure 2D,E). Furthermore, a chromatin immunoprecipitation (ChIP) assay showed an increased affinity of NRF2 to the promoters of $\mathrm{NAD}(\mathrm{P}) \mathrm{H}$ dehydrogenase [quinone] 1 (NQO1), heme oxygenase (decycling) 1 (HMOX1), glutamate-cysteine ligase catalytic subunit (GCLC), GCLM, and SLC7A11 in SDHB ${ }^{K D}$ cells, confirming increased NRF2-mediated antioxidant gene transcription (Figure 2F).

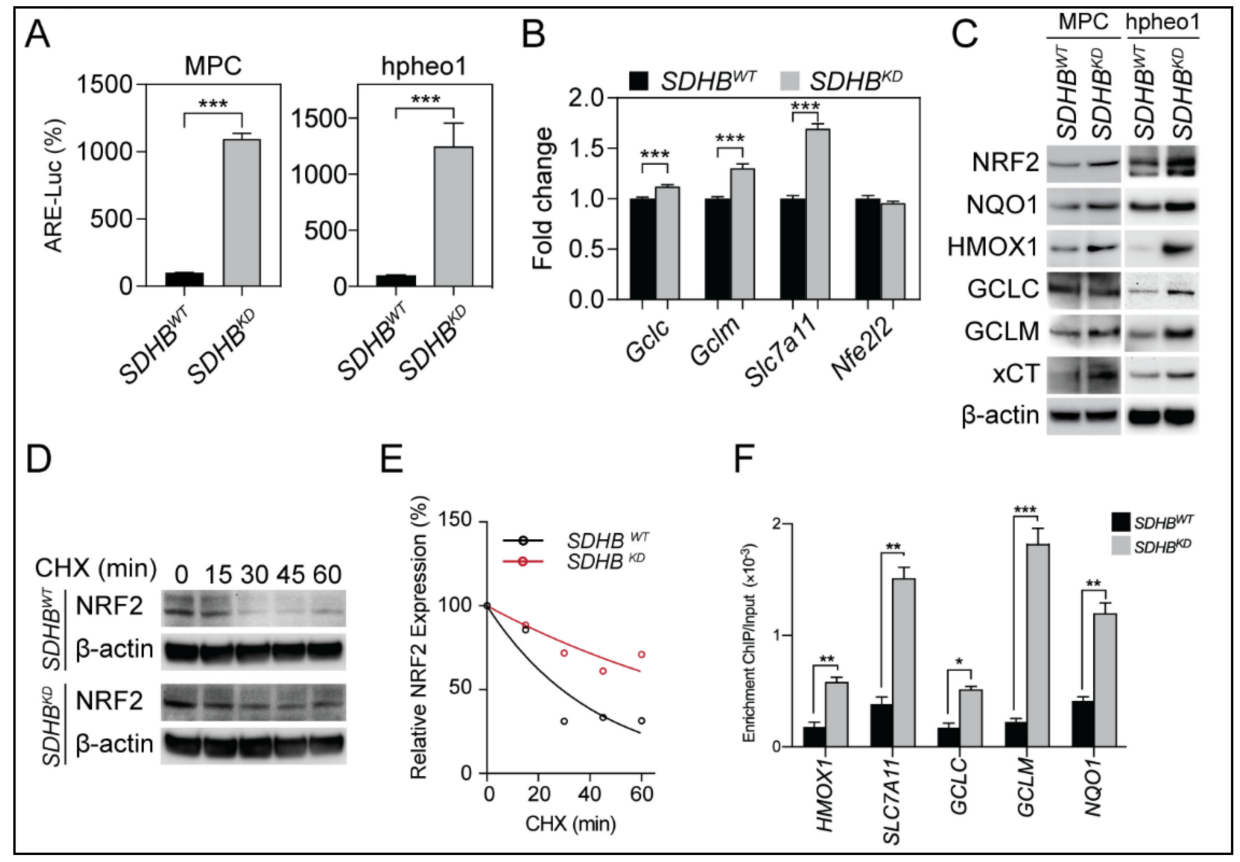

Figure 2. $S D H B$ deficiency activated NRF2-driven glutathione synthetic pathway. (A) antioxidative response element (ARE)-luciferase reporter assay showed increased NRF2 activity in SDHB ${ }^{K D}$ MPC and hpheo1 cells. ${ }^{* *} p<0.001$. (B) Quantitative real-time PCR showed increased gene transcription of NRF2-associated genes Gclm and Slc7a11 in SDHB ${ }^{K D}$ MPC cells. ${ }^{* *} p<0.001$. (C) Immunoblotting showed increased expression of NRF2 and its downstream targets in $S D H B^{K D}$ MPC and hpheo1 cells. $\beta$-actin was used as internal control. (D) Cycloheximide (CHX) pulse chase assay showed elevated NRF2 protein stability in $S D H B^{K D}$ hpheo1 cells. $\beta$-actin was used as internal control. (E) Quantification of NRF2 half-life from Figure 2D. (F) Chromatin immunoprecipitation (ChIP) PCR assay showed increased promoter affinity of NRF2 in $S D H B^{K D}$ hpheo1 cells. ${ }^{*} p<0.05 ;{ }^{* *} p<0.01 ;{ }^{* * *} p<0.001$.

\subsection{Glutathione Synthesis Protected SDHB ${ }^{K D}$ Cells}

The substantial upregulation of glutathione synthesis enzymes indicated the reprogramming of glutathione metabolism in SDHB-deficient cells, which might be essential to maintain cell survival under intense oxidative stress. To evaluate the role of the glutathione synthesis pathway, we designed and investigated small interfering RNA targeting GCLC, GCLM, and SLC7A11 (Figure 3A and Figure $\mathrm{S} 3 \mathrm{~A})$. The quantification of intracellular glutathione showed that the genetic silencing of glutathione synthesis enzymes depleted GSH levels in $S D H B^{K D}$ cells, whereas this effect was not seen in $S D H B^{W T}$ cells (Figure 3B). In addition, we evaluated the role of the glutathione synthesis pathway in cellular proliferation. The Cell Counting Kit-8 (CCK8) assay and direct cell counting showed that suppressing the glutathione synthesis pathway halted cellular proliferation in $S D H B^{K D}$ cells (Figure $3 C, D$ and Figure S3B). Moreover, annexin V/propidium iodide (PI) flow cytometry revealed potent apoptotic changes in $S D H B^{K D}$ cells when the glutathione synthesis pathway was suppressed, whereas this phenomenon was not seen in $S D H B^{W T}$ cells (Figure 3E,F and Figure S3C,D). These data demonstrated that $S D H B$ deficiency established a dependency on enhanced glutathione synthesis, which not only 
relieved the oxidative stress but also supported cellular physiology in oncogenic pathways. This finding also suggests that the glutathione antioxidant pathway could be a valuable therapeutic target for malignancies with $S D H B$ deficiency.

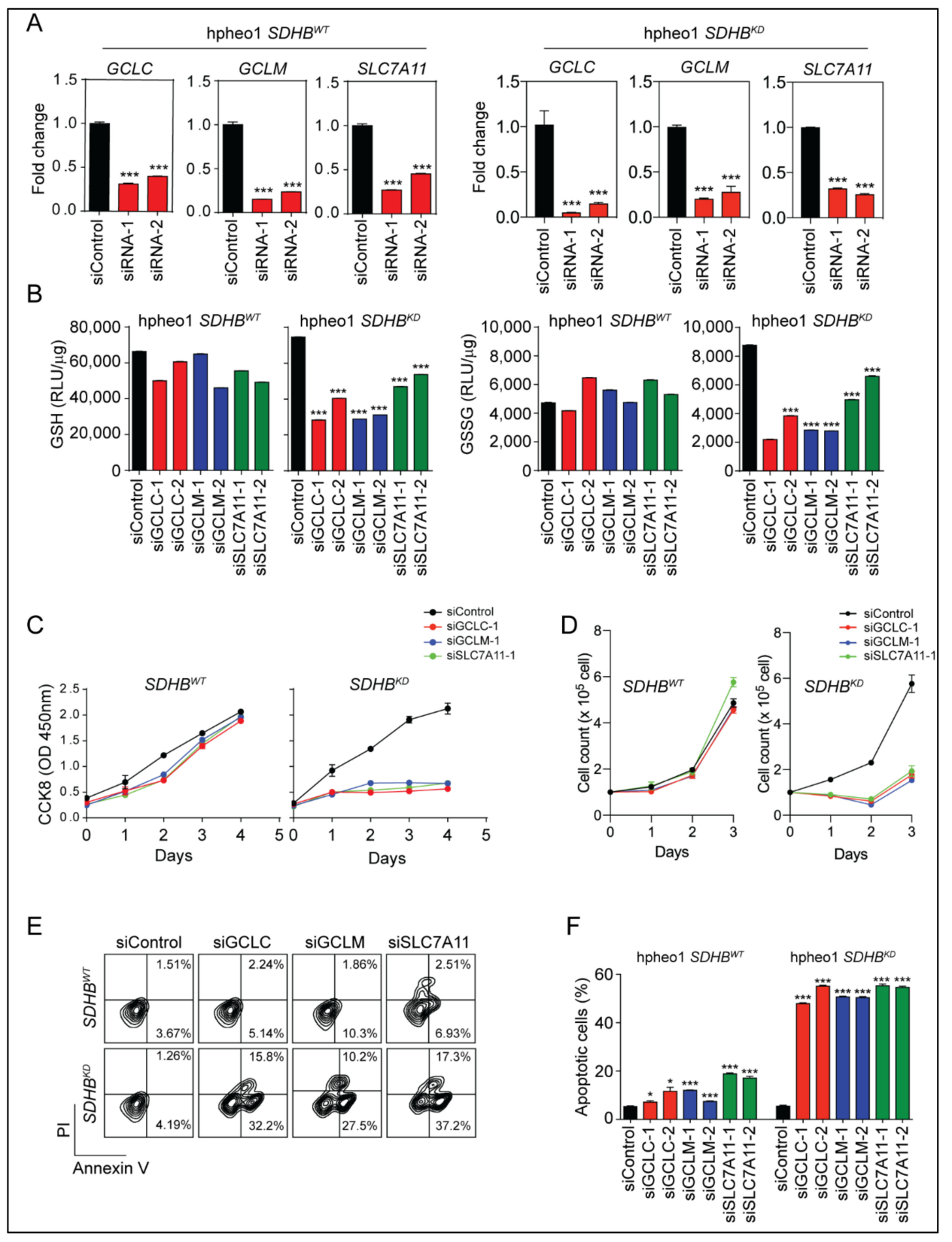

Figure 3. Glutathione synthesis is crucial for the survival and proliferation of $S D H B^{K D}$ cells. (A) Quantitative real-time PCR showed the knockdown efficiency of small interference ribonucleic acid (siRNA) targeting GCLC, GCLM, and SLC7A11 in hpheo1 cells. ${ }^{* * *} p<0.001$. (B) Glutathione quantification assay showed that the cellular GSH and GSSG level was decreased in $S D H B^{K D}$ hpheo1 cells with siRNA targeting GCLC, GCLM, and SLC7A11. ${ }^{* * *} p<0.001$. (C) CCK8 assay showed that the cell viability of $S D H B^{K D}$ hpheo1 cells was suppressed with siRNAs targeting GCLC, GCLM, or SLC7A11. (D) Direct cell count showed reduced cell number of $S D H B^{K D}$ hpheo1 cells with siRNAs targeting GCLC, GCLM, or SLC7A11. (E) Annexin V/PI apoptosis assay showed the apoptotic changes of SDHBKD hpheo1 cells with siRNAs targeting GCLC, GCLM, and SLC7A11. (F) Quantification of apoptosis assay, $S D H B^{K D}$ hpheo1 cells showed increased cell apoptosis. ${ }^{*} p<0.05 ;{ }^{* * *} p<0.001$. 


\subsection{The NRF2 Inhibitor Brusatol Disrupted Glutathione Synthesis}

We investigated whether brusatol could be effective for $S D H B^{K D}$ PCPG cells due to their dependency on antioxidant scavenging. The ARE-luciferase reporter assay showed that brusatol potently eliminated NRF2-derived transcriptional activity in $S D H B^{K D}$ cells (Figure $4 \mathrm{~A}$ ). This finding was confirmed by immunoblotting, evidenced by a reduction in the expression of NRF2, GCLC, GCLM, and xCT upon brusatol treatment (Figure 4B). Accordingly, ChIP PCR showed that the affinity of NRF2 to the promoters of GCLC, GCLM, and SLC7A11 was impaired after brusatol treatment (Figure 4C). In addition, we found that brusatol reduced the half-life of NRF2 protein and prompted NRF2 ubiquitination (Figure S4A-C). The suppression of the glutathione synthesis pathway further reduced the availability of GSH for ROS scavenging, as the GSH/GSSG ratio decreased after brusatol treatment in $S D H B^{K D}$ cells, whereas the ROS scavengers NAC and catalase could restore GSH levels (Figure 4D and Figure S4D). These results indicate that the glutathione synthesis pathway is one of the primary targets within brusatol-induced NRF2 inhibition.

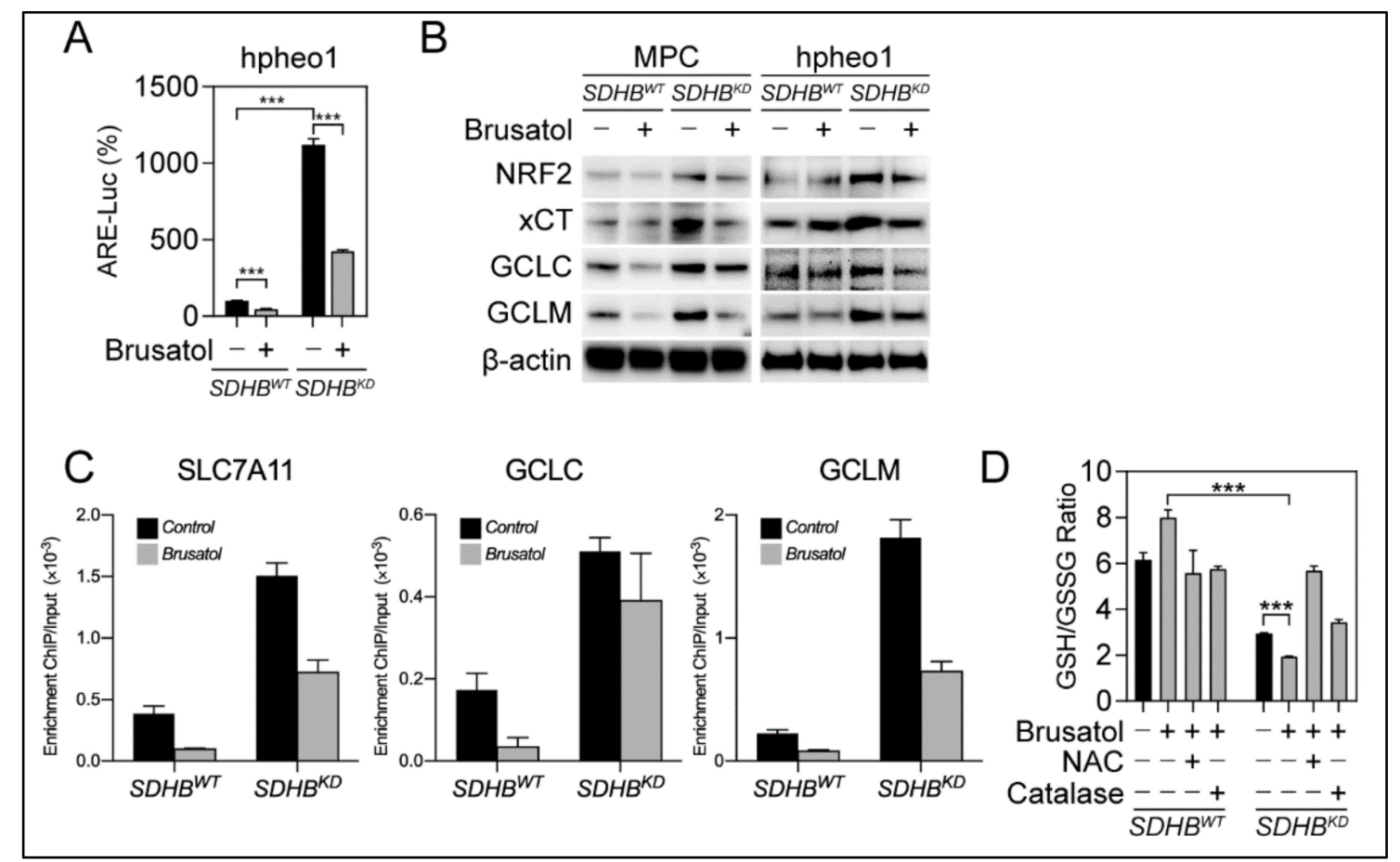

Figure 4. Suppressing of NRF2 activity inhibits glutathione synthesis pathway in $S D H B^{K D}$ cells. (A) ARE-luciferase reporter assay showed brusatol treatment inhibited NRF2 transcriptional activity in $S D H B^{K D}$ hpheo1 cells. ${ }^{* * *} p<0.001$. (B) Immunoblotting showed that brusatol suppressed the expression of NRF2, xCT, GCLC, and GCLM in MPC and hpheo1 cells. $\beta$-actin was used as internal control. (C) ChIP PCR assay showed that Brusatol reduced promoter affinity of NRF2 to SLC7A11, GCLC, and GCLM. (D) Glutathione quantification showed that brusatol reduced GSH/GSSG ratio in $S D H B^{K D}$ MPC cells. Exogenous ROS scavengers NAC and Catalase can restore the ratio with the presence of Brusatol. ${ }^{* * *} p<0.001$.

\subsection{NRF2 Suppression Led to Oxidation-Derived Cellular Damage}

The suppression of the NRF2 antioxidative pathway depleted the intracellular pool of GSH, which may translate into oxidation-derived cellular damage. Both the ROS-Glo and MitoSOX Red staining assays showed that the ROS level remarkably increased after brusatol treatment (Figure 5A-C). High ROS levels in $S D H B^{K D}$ cells resulted in oxidative damage in both mitochondrial and genomic DNA (Figure 5D,E and Figure S6G,H). The comet assay confirmed increased DNA fragmentation in $S D H B^{K D}$ cells treated with brusatol, whereas exogenous ROS scavengers salvaged DNA fragmentation in the 
same assay (Figure 5F,G). Furthermore, DNA oxidative damage ELISA and $\gamma$ H2A.X staining confirmed oxidative DNA damage in $S D H B^{K D}$ cells with brusatol (Figure $5 \mathrm{H}-\mathrm{J}$ ).

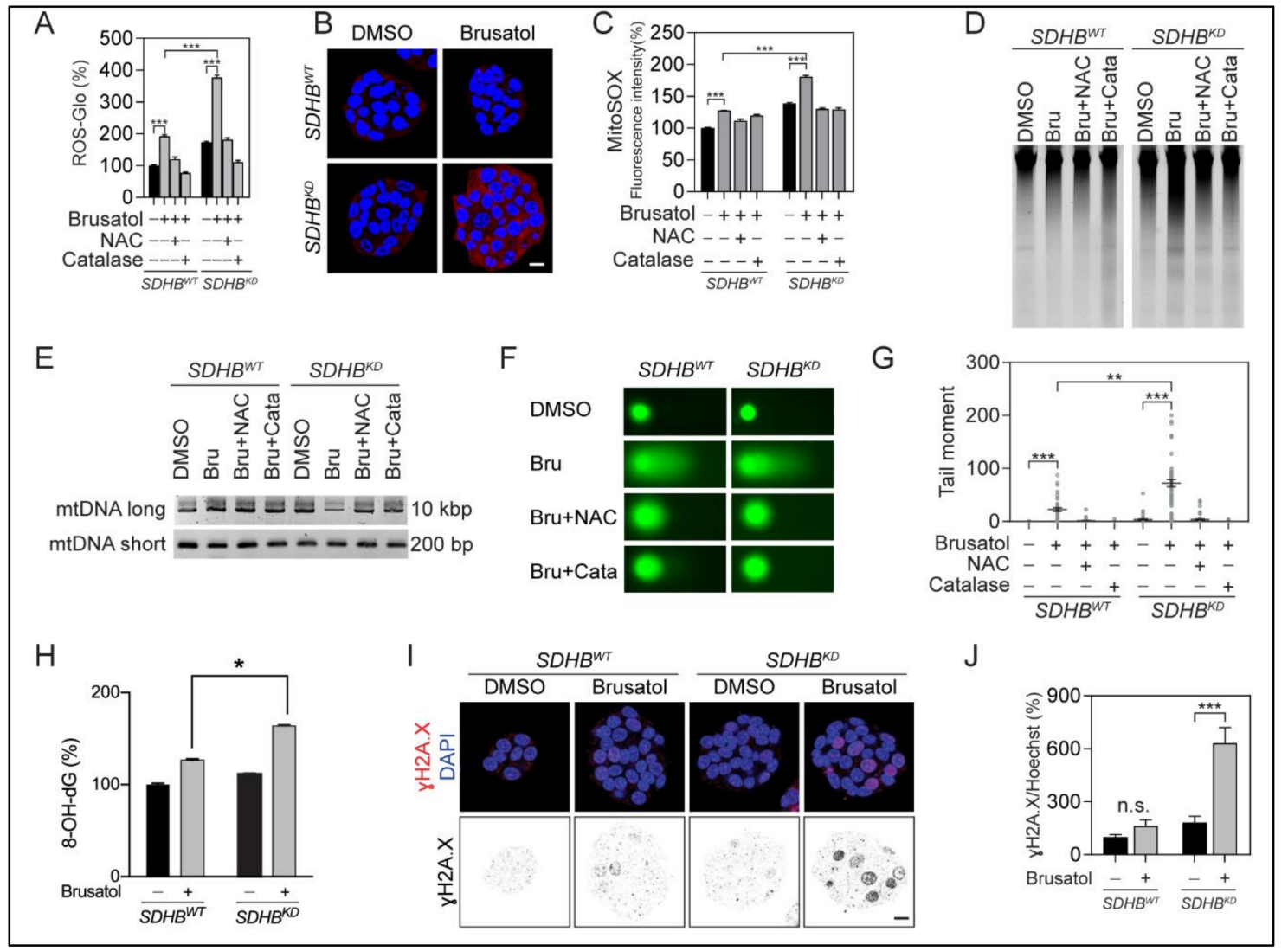

Figure 5. Brusatol disrupted ROS homeostasis and led to oxidative DNA damage. (A) ROS quantification assay showed that brusatol increased ROS level, especially in $S D H B^{K D}$ MPC cells. Exogenous ROS scavengers restored ROS to the normal level. ROS signal was measured and normalized to protein quantification. ${ }^{* * *} p<0.001$. (B) MitoSOX-Red staining showed increased ROS level in $S D H B^{K D}$ MPC cells after brusatol treatment. Bar $=10 \mu \mathrm{m}$. (C) Flowcytometry analysis showed increased MitoSOX-Red signal in $S D H B^{K D}$ cells after brusatol treatment. ROS scavengers restored ROS to normal level. Dimethyl sulfoxide (DMSO) was used as solvent control. Cell nuclei were labeled with 4',6-diamidino-2-phenylindole (DAPI). Bar $=10 \mu \mathrm{m}$. (D) Total genomic DNA electrophoresis showed increased DNA fragmentation in $S D H B^{K D}$ MPC cells with brusatol treatment. (E) Mitochondrial deoxyribonucleic acid (mtDNA) PCR assay showed the long and short fragments from the mitochondrial genome of MPC cells was performed after brusatol treatment. Exogenous ROS scavengers restored DNA fragmentation. (F) Comet assay showed increased DNA fragmentation (Comet tail) in SDHB ${ }^{K D} \mathrm{MPC}$ cells. Exogenous ROS scavengers restored DNA fragmentation. (G) Quantification of comet assay in Figure 5F. ${ }^{* *} p<0.01$; ${ }^{* * *} p<0.001$. (H) Oxidative DNA damage ELISA assay showed stronger elevation of 8-OH-dG in $S D H B^{K D}$ MPC cells with brusatol treatment. ${ }^{*} p<0.05$. (I) Immunostaining showed elevated $\gamma \mathrm{H} 2 \mathrm{~A} . \mathrm{X}$ in $S D H B^{K D} \mathrm{MPC}$ cells with brusatol treatment. Bar $=10 \mu \mathrm{m}$. (J) Quantification of $\gamma$ H2A.X in Figure 5I. ${ }^{* * *} p<0.001$.

\subsection{Selective Vulnerability of SDHB ${ }^{K D}$ Cells to NRF2 Suppression}

As a consequence of elevated oxidative stress, we recorded profound and selective cytotoxicity in $S D H B^{K D}$ cells upon brusatol treatment. A cell viability assay showed $S D H B^{K D}$ cells to be more vulnerable to brusatol treatment with an $\mathrm{IC}_{50}$ of $6.3 \mathrm{nM}$ for $S D H B^{K D}$ cells compared with an $\mathrm{IC}_{50}$ of $19.5 \mathrm{nM}$ for $S D H B^{W T}$ cells (Figure 6A). A 5-bromo-2'-deoxyuridine (BrdU) incorporation assay confirmed that brusatol reduced cell proliferation by $35.3 \%$ in $S D H B^{K D}$ cells, whereas an $18.8 \%$ reduction 
was observed in the wild-type counterpart (Figure 6B,C). Similarly, a long-term colony-formation assay revealed that brusatol significantly reduced the colony count in $S D H B^{K D}$ but not $S D H B^{W T}$ cells (Figure 6D). Importantly, the brusatol-mediated reduction in cell proliferation could be rescued by the ROS scavengers NAC or catalase, suggesting the involvement of an overload of oxidative damage. Furthermore, a caspase 3/7 activity assay showed higher apoptotic changes in SDHB-deficient cells (Figure 6E). An immunoblotting assay confirmed caspase cleavage, accompanied with PARP cleavage and $\gamma$ H2A.X elevation in brusatol-treated $S D H B^{K D}$ cells (Figure $6 \mathrm{~F}$ ). In agreement with these findings, annexin V/PI flow cytometry showed an increased number of apoptotic cells in $S D H B^{K D}$ cells compared with $S D H B^{W T}$ after receiving the same dosage of brusatol (Figure $6 \mathrm{G}, \mathrm{H}$ ). The combination with the ROS scavengers NAC and catalase significantly reduced brusatol-induced cytotoxicity, indicating that ROS imbalance is the primary cause of growth arrest and apoptotic changes.

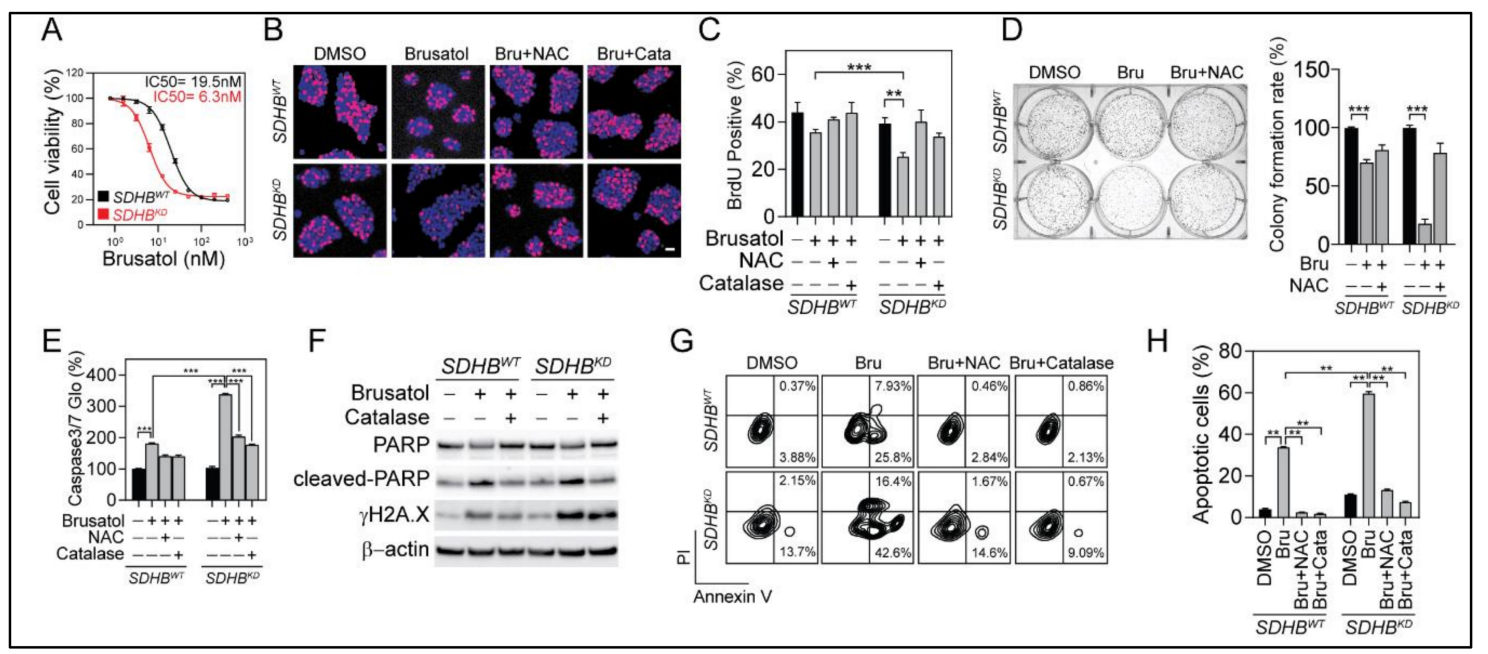

Figure 6. Brusatol selectively suppressed $S D H B^{K D}$ cells. (A) Dose-response curve showed that $S D H B^{K D}$ MPC cells were more vulnerable to Brusatol treatment. (B) BrdU (red) incorporation assay shows that Brusatol selectively inhibited the proliferation of $S D H B^{K D}$ MPC cells. Cell nuclei were labeled with Hoechst 33342 (blue). Bar $=20 \mu \mathrm{m}$. (C) Quantification of BrdU-positive cells in Figure 6B. ${ }^{* *} p<0.01$; $* * * p<0.001$. (D) Long-term colony formation assay and quantification showed that Brusatol inhibited cluster formation of $S D H B^{K D}$ MPC cells. ${ }^{* * *} p<0.001$. (E) Caspase 3/7-Glo assay showed significantly increased Caspase 3/7 activity in $S D H B^{K D}$ MPC cells with Brusatol. Luminescence was measured and normalized to protein quantification. ${ }^{* *} p<0.001$. (F) Immunoblotting showed increased cleaved-poly (ADP-ribose) polymerase (PARP) and $\gamma \mathrm{H} 2 \mathrm{~A} . \mathrm{X}$ after Brusatol treatment. $\beta$-actin was used as internal control. (G) Annexin V/PI apoptosis analysis showed that $S D H B^{K D}$ MPC cells exhibited more apoptotic cells under Brusatol treatment compared with $S D H B^{W T}$ cells. ROS scavengers reduced the number of apoptotic cells. (H) Quantification of apoptotic cells from Figure 6G. ${ }^{* *} p<0.01$.

\subsection{NRF2 Blockade for SDHB-Mutated PCPGs}

Considering the central roles in maintaining ROS homeostasis for $S D H B^{K D}$ cells, targeting the NRF2 antioxidant pathway could be a valuable therapeutic strategy as a selective treatment for SDHB-deficient malignancies. To test this hypothesis, we investigated the tumor-suppressing effect of brusatol in a preclinical animal model bearing metastatic PCPG allografts. We established a liver metastasis model by tail vein injection of MPC $S D H B^{K D}$ cells in NU/J mice. Mice were randomized and administered $1 \mathrm{mg} \mathrm{kg}^{-1}$ brusatol intraperitoneal (i.p.) every other day (Figure 7A). In vivo luminescence imaging showed that brusatol significantly reduced the speed of tumor metastasis (Figure 7B,C). Moreover, brusatol improved disease outcome with significantly prolonged overall survival by $36.1 \%$ ( 36 days versus 49 days, Figure 7D). Furthermore, the immunoblotting assay showed that brusatol suppressed the expression of NRF2 and $\mathrm{xCT}$ in the metastatic lesions (Figure 7E). Consistent with this, immunohistochemistry showed that the expression levels of NRF2, as well as the glutathione metabolic 
enzymes GCLM and xCT, were diminished in the brusatol-treated group (Figure 7F). In addition, low expression of the proliferation marker Ki67 but higher expression of the DNA damage marker $\gamma \mathrm{H} 2 \mathrm{~A} . \mathrm{X} /$ Terminal deoxynucleotidyl transferase dUTP nick end labeling (TUNEL) were observed in brusatol-treated tissue specimens (Figure 7G). However, for animals bearing MPC SDHB ${ }^{W T}$ liver metastasis, brusatol did not effectively suppressed tumor growth, as on the SDHB-deficient cells (Figure S5A,B). The median survival day was slightly extended from 38.5 days to 43 days $(p=0.3424$; Figure S5C). Taken together, these findings demonstrated that SDHB-deficient PCPG cells are sensitive to oxidative stress induced by suppressing NRF2 activity.

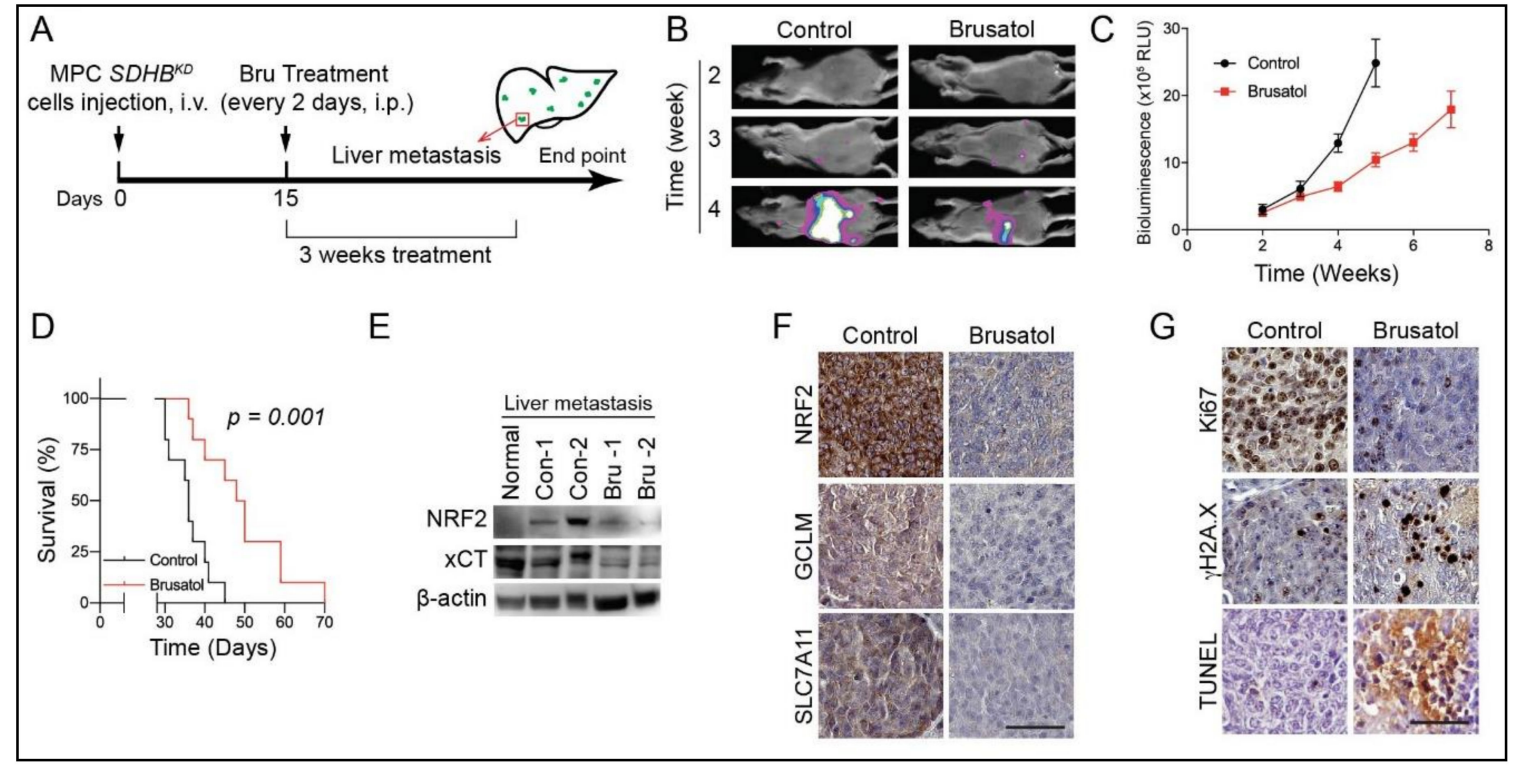

Figure 7. Brusatol suppressed $S D H B$-mutated MPC allograft in vivo. (A) Schematic illustration for the allograft and treatment schedule. (B) Luciferase imaging showed that brusatol treatment suppressed $S D H B^{K D}$ hepatic lesions in vivo. (C) Quantification of tumor volume shown in Figure 7B. (D) Kaplan-Meier analysis showed that brusatol prolonged overall survival of tumor-bearing animal $(p=0.001)$. (E) Immunoblotting showed that Brusatol inhibited the expression of NRF2 and xCT hepatic metastatic lesions. $\beta$-actin was used as internal control. (F) Immunohistochemistry assay showed that the expression of NRF2, xCT, and GCLM were suppressed in metastatic lesions under Brusatol treatment. Bar $=50 \mu \mathrm{m}$. (G) Immunohistochemistry assay showed the staining of Ki67, $\gamma \mathrm{H} 2 \mathrm{~A} . \mathrm{X}$, and TUNEL assay in tumor sections. Bar $=50 \mu \mathrm{m}$.

\section{Discussion}

In the present study, we demonstrated that cluster I PCPG with SDHB deficiency exhibited a unique metabolic pattern, characterized by a high ROS burden and dependency on de novo glutathione synthesis. We showed that $S D H B^{K D}$ cells developed a dependency on NRF2 anti-oxidative pathways to meet the increased needs of ROS detoxification to avoid cell death. NRF2 stabilization and transactivation supported de novo glutathione synthesis through the upregulation of GCLC, GCLM, and SLC7A11. Targeting the NRF2/glutathione axis resulted in ROS overload, oxidative DNA damage, reduced cellular proliferation, and profound cytotoxicity. Moreover, brusatol, a potent NRF2 inhibitor, established synthetic lethality with $S D H B$ deficiency, which significantly improved disease outcome with prolonged overall survival in vivo. Our findings highlight the important role of the NRF2/glutathione axis in cluster I PCPG with SDHB deficiency and a potential therapeutic approach for these and other $S D H B$-related tumors.

$S D H B$ is the iron-sulfur subunit of mitochondrial complex II, which plays a critical role in succinate dehydrogenase activity. Pathogenic mutations in SDHB result in abnormalities in electron transfer steps within mitochondrial complex II, which compromise the citric acid cycle and establish a 
pseudohypoxia phenotype, Warburg-like metabolism, and the formation of several types of human malignancies $[14,29,30]$. Moreover, the loss of the SDHB subunit prompts the accumulation of electrons in the flavin group in SDHA, which promotes superoxide generation through the autoxidation of the reduced flavin group by $\mathrm{O}_{2}$ in the matrix [25]. In the present study, we confirmed that the accumulation of cellular ROS is closely related to the dysfunction of SDHB (Figure 1A-D). The glutathione synthesis pathway was activated as a consequence of elevated intracellular ROS (Figure 1E-H). NRF2, the master transcriptional factor for redox regulation, was stabilized and initiated the transcription of antioxidant genes such as NQO1, HMOX1, GLCL, GCLM, and SLC7A11 (Figure 2). Importantly, we discovered that $S D H B$-mutated cells exhibited a dependency on glutathione consumption, as genetic silencing of glutathione synthesis enzymes compromised cellular viability specifically in cells with SDHB reduction (Figure 3). These findings highlight a metabolic signature in SDHB-mutated PCPG, with markedly enhanced ROS production and scavenging.

Owing to highly harmful oxidative-derived cellular damage, the intracellular ROS level is strictly controlled to a minimal level to protect macromolecules such as DNA, proteins, and lipids. Several evolutionarily conserved mechanisms have been identified to eliminate ROS from cellular compartments. For example, superoxide dismutases (SODs) are enzymes that catalyze the superoxide radical $\left(\mathrm{O}_{2}^{-}\right)$into oxygen $\left(\mathrm{O}_{2}\right)$ or hydrogen peroxide $\left(\mathrm{H}_{2} \mathrm{O}_{2}\right)$. Glutathione peroxidases (GPXs) are a family of enzymes that transform $\mathrm{H}_{2} \mathrm{O}_{2}$ into water in a glutathione/reduced form nicotinamide adenine dinucleotide phosphate (NADPH)-dependent manner [31,32]. The transcription level of these antioxidant genes is regulated by NRF2, which governs the production and scavenging of intracellular ROS [33,34]. ROS accumulation in the cytoplasm compromises the function of kelch-like ECH-associated protein 1 (KEAP1), the E3 ligase that deactivates NRF2, leading to the stabilization and functioning of NRF2 to activate the antioxidant pathway [23,34,35]. In the present study, we confirmed that in cells with $S D H B$ deficiency, the high ROS levels are sufficient to stabilize NRF2 (Figure 2D,E). NRF2 translocates into the nucleus and mediates the transcriptional activation of several key antioxidant genes such as GCLC, GCLM, and SLC7A11 (Figure 2F). The enhancement of the glutathione synthesis pathway was confirmed in patient-derived specimens, as the enzymes were upregulated in SDHB-defective cluster I PCPG (Figure 1F-H). These findings highlight that the NRF2-derived glutathione synthesis pathway plays a critical role in SDHB-deficient malignancies, which is a key clue to the therapeutic vulnerability for this disease cluster.

In the present study, the NRF2-derived de novo glutathione synthesis pathway was targeted as a therapeutic approach for PCPGs with SDHB deficiency. Brusatol has been repeatedly shown to be a potent inhibitor for NRF2 and antioxidant pathways [24,36-39]. Several studies have indicated that brusatol alone exhibits mild cytotoxic effects and is frequently evaluated as a sensitization approach to support other cytotoxic therapies such as radiation therapy and cisplatin $[24,40,41]$. Interestingly, our in vitro assays and preclinical animal models showed that brusatol effectively suppressed $S D H B$-mutated malignancies as a single therapeutic agent (Figures 5 and 6 ). We believe that in malignancies with intrinsic metabolic deficiencies, such as SDHB-mutated PCPG, elevated cytosolic ROS synergizes with antioxidant pathway inhibition. The synthetic lethality approach is sufficient in causing overwhelming oxidative-derived damage in mitochondrial and genomic DNA, which proceeds to apoptotic changes and cancer suppression.

\section{Materials and Methods}

\subsection{Study Design}

This study was designed to evaluate the role of NRF2-govern glutathione synthesis axis in SDHB-deficient PCPG and explore potential therapeutic targets. In this study, we tested our hypothesis on the basis of two previously reported PCPG models: mouse pheochromocytoma (MPC) and human pheochromocytoma (hpheo1) cells, through both in vitro and in vivo assays. Clinical samples from patients with cluster I and II PCPG were used as validation. All experiments were replicated three 
times, and the key experiments were repeated in two different cell line models. The investigators were not blinded, and data were collected and analyzed objectively.

\subsection{Patient Samples}

Tumor samples from cluster I and cluster II PCPG patients were dissected from the clinical specimens. This study was approved by the Institutional Review Board of the Eunice Kennedy Shriver National Institute of Child Health and Human Development (NICHD), National Institutes of Health (NIH), and all patients gave written informed consent. The IRB Protocol \#: 00CH0093.

\subsection{Cell Lines}

Mouse pheochromocytoma (MPC) cell lines were cultured in Dulbecco's modified Eagle's medium (DMEM) containing 10\% fetal bovine serum (FBS), penicillin, and streptomycin (Thermo Fisher, Waltham, MA, USA). $S D H B$ wild type $\left(S D H B^{W T}\right)$ and $S D H B$ knock down $\left(S D H B^{K D}\right)$ MPC cells were described previously [20]. Progenitor cell line derived from human pheochromocytoma (hpheo1) was a gift from Dr. Hans Ghayee approved under material transfer agreement, Eunice Kennedy Shriver NICHD [42]. SDHB-deficient hpheo1 was prepared by lentivirus with short hairpin RNA targeting SDHB (V3SVHS00_8112304, targeting sequence CAG AGC TGA ACA TAA TTT A, GE Dharmacon, Boston, MA, USA). Puromycin selection was performed to establish isogenic cells, and knockdown efficiency was confirmed by immunoblotting.

\subsection{Reagents and Treatment Condition}

Brusatol, $N$-acetylcysteine (NAC) and catalase were purchased from Sigma (St. Louis, MO, USA) and dissolved in phosphate-buffered saline (PBS). The final concentration used in the present study was Brusatol $40 \mathrm{nM}$, NAC $2.5 \mathrm{mM}$, and catalase $500 \mathrm{U} \mathrm{mL}^{-1}$. For gene expression analysis and oxidative stress and DNA damage-related experiments, cells were treated with indicated conditions for $24 \mathrm{~h}$. For cytotoxicity related experiments, cells were under treatment for $72 \mathrm{~h}$.

\subsection{Real-Time PCR}

Total RNA was extracted from MPC and hpheo1 cells using PureLink RNA mini kit (Thermo Fisher), and reverse transcript to DNA using Superscript IV VILO Master Mix (Thermo Fisher). Real-time PCR was performed using Power SYBR Green Master Mix. Primers used in the current study included: mGCLC.F: 5'-CTA CCA CGC AGT CAA GGA CC-3'; mGCLC.R: 5' -CCT CCA TTC AGT AAC AAC TGG AC-3'; mGCLM.F: 5'-ACT CAC AAT GAC CCG AAA GAA C-3'; mGCLM.R: 5'-CCT GCT CTT CAC GAT GAC CG-3'; mSLC7A11.F: 5'-GGC ACC GTC ATC GGA TCA G-3'; mSLC7A11.R: 5'-CTC CAC AGG CAG ACC AGA AAA-3'; hNQO1 (QT00050281); hHMOX1 (QT00092645); hNFE2L2 (QT00027384); hSLC7A11 (QT00002674); and hACTB (QT00095431).

\subsection{RNA Interference}

Small interference RNA (siRNA) oligos were designed and synthesized by Integrated DNA Technologies (IDT, Coralville, IA, USA). The sequences of siRNA used in the study are listed as follows: siGCLC.1.F: 5'-ACA AUU GGA CAG AUA GUA GCC AAC UGA-3'; siGCLC.1.R: 5' -AGU UGG CUA CUA UCU GUC CAA UUG T-3'; ; siGCLC.2.F: $5^{\prime}$-UAA AUA UUG GUA CAU UGA UGA CAA CCU-3'; siGCLC.2.R: $5^{\prime}$-GUU GUC AUC AAU GUA CCA AUA UUT A-3'; siGCLM.1.F: 5'-AAG GUU UUU UGG AUA CAA UCA UGA AGC-3'; siGCLM.1R: $5^{\prime}$-UUC AUG AUU GUA UCC AAA AAA CCT T-3'; siGCLM.2.F: 5'-CCU UCU UUU AGC UUG UAA AAU GUA GCC-3'; siGCLM.2.R: 5' -CUA CAU UUU ACA AGC UAA AAG AAG G-3'; siSLC7A11.1.F: 5'-AUG ACU GUG CUU CCA AGU AUG CAU CUA-3'; siSLC7A11.1.R: 5'-GAU GCA UAC UUG GAA GCA CAG UCA T-3'; siSLC7A11.2.F: 5'-UUC UUU AUA GUU GUU CCC AAU UCA GCA-3'; siSLC7A11.2.R: 5'-CUG AAU UGG GAA CAA CUA UAA AGA A-3'. AllStar negative control siRNA (QIAGEN, Hilden, Germany) was used as a control. 


\subsection{Chromatin Immunoprecipitation (ChIP) Assay}

The ChIP assay was performed by using a ChIP-IT High Sensitivity kit (Active Motif, Carlsbad, CA, USA) according to the manufacturer's protocol. A total of 15 million cells were fixed and collected for nuclei isolation. Chromatin was prepared from isolated nuclei, sheared by sonication, and precipitated with anti-NRF2 antibody (Active Motif). Promoter enrichment was quantified by quantitative real-time PCR assay comparing pull-down DNA and input DNA. The sequences of the primer set used are listed as follows: HMOX1.F: 5' -ACA AAG GGA AGG CGG ATT TT-3'; HMOX1.R: 5' -ACT TCC TCC TGC CTA CCA TT-3'; SLC7A11.F: 5'-AGC TTC CCA CAA AGT CGA AG-3'; SLC7A11.R: 5'-ACA TTC CTG CTT GTC TTG GT-3'; GCLC.F: 5'-CGC AGT TGT TGT GAT ACA GCC-3'; GCLC.R: 5'-GGA CTG AGA CTT TGC CCT AAG AA-3'; GCLM.F: 5'-ATT CCA AAC TGA GGG AGC TGT TT-3'; ; $5^{\prime}$-GCLM.R: ATG AGT AAC GGT TAC GAA GCA CT-3'; NQO1.F: 5' -GTG TGA CAG AGG CCT CAA AA-3'; NQO1.R: 5'-TGA TCC CTG GAC TCT CTT GG-3'.

\subsection{ROS Quantification}

Cellular ROS was measured using ROS-Glo $\mathrm{H}_{2} \mathrm{O}_{2}$ assay kit (Promega, Madison, WI, USA) according to the manufacturer's protocol. The luminescence signal was measured using Polarstar Optima plate reader (BMG LABTECH, Ortenberg, Germany). Mitochondrial ROS were measured using MitoSOX staining (Thermo Fisher). Cells were incubated with $5 \mu \mathrm{M}$ MitoSOX-Red at $37^{\circ} \mathrm{C}$ for $10 \mathrm{~min}$ and analyzed by either confocal imaging using Zeiss 710 NLO or flow cytometry using LSRFortessa SORP or FACSCanto II. Fluorescence intensity was measured and quantified by using ImageJ software (v1.8.0_112).

\subsection{ARE Luciferase Reporter Assay}

ARE transcriptional activity was determined using either transfection of reporter plasmid pGL4.37-luc2P-ARE-Hygro (Promega, Madison, WI, USA) or Cignal Antioxidant Response Reporter system (Qiagen) according to the manufacturer's protocol. For dual luciferase assay, $900 \mathrm{ng}$ pGL3.47-ARE-Luc and $100 \mathrm{ng}$ pRL-TK (Promega) were transfected into 10,000 cells using Lipofectamine 2000 (Thermal Fisher, Waltham, MA, USA). Luminescence signal was recorded using Polarstar Optima plate reader and normalized to protein quantification or Renilla luciferase activity.

\subsection{Immunoblotting}

Total protein was extracted from cultured cells using RIPA lysis buffer supplemented with protease and phosphatase inhibitor cocktail (Thermo Fisher). The cell lysates were resolved on Bis-Tris gel (Thermo Fisher) and transferred to polyvinylidene difluoride (PVDF) membrane (Millipore, Burlington, MA, USA). After blocking in Superblock (Thermo Fisher), the membrane was incubated with primary antibodies at $4{ }^{\circ} \mathrm{C}$ overnight. Protein was further probed by horseradish peroxidase (HRP)-conjugated secondary antibodies and visualized using a Bio-Rad ChemiDoc Imaging System. The antibodies used in the present study included: NRF2 (CST, Danvers, MA, 1:1000), GCLC (Abcam, Cambridge, United Kingdom, 1:1000), GCLM (Proteintech, Rosemont, IL, 1:1000), xCT (Abcam, 1:1000), NQO1 (Abcam, 1:1000), Ubiquitin (Abcam, 1:2000), PARP1 (CST, 1:1000), $\gamma$ H2A.X (CST, 1:1000), EGFP (Thermo Fisher, 1:1000), Human influenza hemagglutinin (HA)-tag (Covance, Princeton, NJ, 1:1000), DYKDDDDK peptide tag (DDK, Origene, Rockville, MD, 1:2000), and $\beta$-actin (Sigma, 1:5000). All immunoblotting images are shown in Figure S6.

\subsection{Immunofluorescence Staining}

Cells were seeded in 8-well chamber slides (Ibidi, Martinsried, Germany), fixed in 4\% paraformaldehyde and permeabilized with $0.3 \%$ TritonX-100. Cells were blocked in Superblock (Thermo Fisher) and incubated with $\gamma \mathrm{H} 2 \mathrm{~A} . \mathrm{X}$ antibody (CST, 1:200) at $4{ }^{\circ} \mathrm{C}$ overnight. Cells were 
washed three times with PBS and incubated with fluorescent conjugated secondary antibody (Thermo Fisher). Images were taken using a Zeiss LSM710 confocal microscope (Oberkochen, Germany).

\subsection{Annexin V/PI Apoptosis Assay}

Cellular apoptosis was analyzed by Annexin V/PI apoptosis kit (Thermo Fisher) according to the manufacturer's manual. Cells were lifted by trypsin and stained with annexin V-Alexa 488 and PI in binding buffer for 20 min on ice. Fluorescent signal was analyzed using a FACS Canto II flow cytometer (BD, Franklin Lakes, NJ, USA).

\subsection{Caspase-3/7 Activity Assay}

A total of 5000 cells were seeded in a 96-well plate and caspase 3/7 activity was measured using a Caspase-Glo 3/7 assay (Promega) according to the manufacturer's manual. Luminescence signal was measured by a Polarstar Optima plate reader and normalized to protein quantification.

\subsection{DNA Oxidative Damage ELISA Assay}

DNA oxidative damage was measured using Cayman DNA/RNA Oxidative Damage ELISA kit (Cayman Chemical, Ann Arbor, MI, USA), according to the manufacturer's protocol. Total DNA was extracted from cultured cells and digested using nuclease P1. A total of 100 ng of DNA was loaded into a microwell plate and DNA/RNA oxidative damage was measured by ELISA assay.

\subsection{DNA Fragmentation Assay}

Cells were harvested and total DNA was isolated and purified using QIAamp DNA Blood kit (QIAGEN). Then, $500 \mathrm{ng}$ of DNA was resolved by electrophoresis using 4-20\% Tris-borate-EDTA (TBE) gel (Thermo Fisher). Gel was stained with SYBR Safe (Thermo Fisher) and exposure using a Bio-Rad ChemiDoc Imaging System. Mitochondrial DNA damage was measured as previously described [43]. A long fragment $(10.1 \mathrm{~kb})$ of mitochondrial DNA (mtDNA) was amplified using primers mtDNA.long.F: 5'-GCC AGC CTG ACC CAT AGC CAT AAT AT-3' and mtDNA.long.R: $5^{\prime}$-GAG AGA TTT TAT GGG TGT AAT GCG G-3'. A short fragment (241 bp) mtDNA was amplified with the primers mtDNA.short.F: 5'-CCT CCC ATT CAT TAT CGC CGC CCT TGC-3' and mtDNA.short.R: 5' -GTC TGG GTC TCC TAG TAG GTC TGG GAA-3’.

\subsection{Comet Assay}

Alkaline Comet assay was performed to evaluate the DNA damage as we described before [44]. Cells were harvested, diluted in low temperature melting agarose, and spread on pre-coated glass slides. After lysis, the slides were subjected to electrophoresis (voltage: $1 \mathrm{~V} \mathrm{~cm}^{-1}$ ) for $1 \mathrm{~h}$ at room temperature in dark. Each slide was stained with SYBR Green and evaluated by confocal microscopy.

\subsection{BrdU-Incorporated Cell Proliferation}

BrdU cell proliferation assay was performed as previous described [20]. Generally, cells were incubated with $10 \mu \mathrm{M}$ of BrdU for $2 \mathrm{~h}$ and fixed in $4 \%$ paraformaldehyde (PFA). Cells were then treated with $2 \mathrm{~N} \mathrm{HCl}$ and neutralized with $0.1 \mathrm{M}$ sodium borate. Cells were stained with mouse anti-BrdU antibody ( $0.5 \mu \mathrm{g}$ per test, BD Biosciences) overnight at $4{ }^{\circ} \mathrm{C}$. After staining with donkey anti-mouse immunoglobulin G (IgG) Alexa Fluor 488, fluorescent signal was recorded by confocal microscopy.

\subsection{Colony Formation Assay}

$S D H B^{W T}$ and $S D H B^{K D}$ MPC cells were seeded in a 6-well plate at a density of 1000 cells per well. Cells were treated with compounds for 2 weeks, fixed in $4 \%$ cold formaldehyde (Sigma), and stained with $2 \%$ crystal violet (Sigma). This experiment was repeated by three replicates, and the images were quantified by ImageJ. 


\subsection{Cell Viability Analysis}

The cell counting kit-8 (CCK8, Dojindo, Rockville, MD, USA) was performed to determine cell viability. CCK8 was added at a ratio of 1:20 to cell media and incubated at $37^{\circ} \mathrm{C}$ for $1 \mathrm{~h}$. The absorbance of optical density (OD) $450 \mathrm{~nm}$ was measured by an Epoch plate reader (BioTek, Winooski, VT, USA). Each sample was done in triplicate and results are presented as mean \pm standard error of mean (SEM).

\subsection{Immunoprecipitation and Ubiquitination Assay}

Immunoprecipitation was performed as previously described [45]. NRF2-myc-DDK and Ubiquitin-HA plasmids were transfected into hpheo1 $S D H B^{W T}$ and $S D H B^{K D}$ cells using lipofectamine 3000 (Thermo Fisher). Cells were harvested and lysed in ice-cold RIPA buffer supplemented with protease/phosphatase inhibitor cocktail (Thermo Fisher) and 1\% SDS. NRF2 was pulled down by protein G Dynabeads (Thermo Fisher) and antibody against DDK. Bounded protein was eluted and analyzed by Western blot.

\subsection{CHX Pulse Chase Protein Half-Life Measurement}

CHX pulse chase assay was performed as previously described [46]. NRF2-EGFP plasmid was transfected into hpheo1 $S D H B^{W T}$ and $S D H B^{K D}$ cells. Cells were treated with brusatol overnight. Cells were exposed to $200 \mu \mathrm{g} \mathrm{mL}{ }^{-1} \mathrm{CHX}$ (Sigma). Samples were collected at different time points and analyzed by Western blot.

\subsection{Metastatic Allograft Mouse Model}

The animal experiments were conducted with the principles and procedures outlined in the Eunice Kennedy Shriver NICHD Guide for the Care and Use of Animals and approved by the Animal Care and Use Committee of the National Institute of Health (Animal Study Protocol: 15-028). MPC SDHB ${ }^{K D}$ Luc cells (1.5 million) suspended in normal phosphate-buffered saline (PBS) solution were injected into the tail vein of female athymic mice (Ncr-nu/nu, aged 8 weeks). Ten days after inoculation, animals were screened by IVIS imaging system to evaluate the presence of metastatic lesions. Mice were randomized into two groups (10 mice per group) and treated with PBS or brusatol. Brusatol was injected intraperitoneal (i.p.) every other day at $1 \mathrm{mg} \mathrm{kg}^{-1}$. All animals were carefully monitored every day and tumor growth was recorded weekly by IVIS in vivo imaging system. At the end of the experiments, all animals were sacrificed, and liver metastatic lesions were harvested for further analysis [47].

\subsection{Immunohistochemistry and TUNEL Assay}

Immunohistochemistry was performed in tumor sections, as reported previously [20]. Formalin-fixed, paraffin-embedded (FFPE) tissue slides were incubated with NRF2 (Abcam, ab62352), xCT (Abcam, ab37185), GCLM (Proteintech, 14241-1-AP), and Ki67 (Abcam, ab15580) antibodies and followed with 3,39-diaminobenzidine (DAB) substrate. Slides were counter-stained by hematoxylin and visualized by light microscope.

\subsection{Statistical Analysis}

Statistical analysis was performed using Student's $t$-Test between two groups. Differences among groups were analyzed using one-way ANOVA test followed by Student's $t$-Test as the post-statistical analysis. All tests were two-sided, the results were presented as mean \pm SEM. A $p$-value $<0.05$ was considered as statistically significant. All of the analysis was conducted using GraphPad Prism 7.01 (GraphPad Software, San Diego, CA, USA). 


\section{Conclusions}

In summary, we demonstrated that SDHB-deficient cluster I PCPG exhibited high ROS generation, leading to a dependency on NRF2-driven de novo glutathione synthesis. Targeting the anti-oxidative pathways of the master transcription factor NRF2 could be a new approach to treat this type of cancer through synthetic lethality with intrinsic ROS burden.

Supplementary Materials: The following are available online at http://www.mdpi.com/2072-6694/12/2/280/s1, Figure S1: Metabolic characterization of SDHB-deficient cells, Figure S2: Ectopic expression of SDHB restored redox balance in SDHB-deficient cells, Figure S3: Glutathione synthesis is crucial for the survival in SDHB-deficient MPC cells, Figure S4: Brusatol induced NRF2 ubiquitination and degradation and suppressed glutathione synthesis, Figure S5: Brusatol treatment in SDHB wild-type MPC allograft in vivo, Figure S6: Western Blot and DNA electrophoresis images.

Author Contributions: Y.L. and Y.P. supervised and performed the experiments and wrote the manuscript; V.C., D.Y., and Y.Z. helped with the experiments and animal modelling; T.-T.H., H.G., and J.D. helped with data analysis; K.P. and C.Y. designed and supervised the experiments and contributed towards writing the manuscript, with input from all authors. All authors have read and agreed to the published version of the manuscript.

Funding: This research received no external funding.

Acknowledgments: This research was supported by the Intramural Research Program of the Center for Cancer Research, National Cancer Institute and the Eunice Kennedy Shriver National Institute of Child Health and Human Development.

Conflicts of Interest: The authors declare no conflict of interest.

\section{References}

1. Castro-Vega, L.J.; Letouze, E.; Burnichon, N.; Buffet, A.; Disderot, P.H.; Khalifa, E.; Loriot, C.; Elarouci, N.; Morin, A.; Menara, M.; et al. Multi-omics analysis defines core genomic alterations in pheochromocytomas and paragangliomas. Nat. Commun. 2015, 6, 6044. [CrossRef] [PubMed]

2. Burnichon, N.; Vescovo, L.; Amar, L.; Libe, R.; de Reynies, A.; Venisse, A.; Jouanno, E.; Laurendeau, I.; Parfait, B.; Bertherat, J.; et al. Integrative genomic analysis reveals somatic mutations in pheochromocytoma and paraganglioma. Hum. Mol. Genet. 2011, 20, 3974-3985. [CrossRef] [PubMed]

3. Pang, Y.; Liu, Y.; Pacak, K.; Yang, C. Pheochromocytomas and Paragangliomas: From Genetic Diversity to Targeted Therapies. Cancers 2019, 11, 436. [CrossRef] [PubMed]

4. Bausch, B.; Borozdin, W.; Mautner, V.F.; Hoffmann, M.M.; Boehm, D.; Robledo, M.; Cascon, A.; Harenberg, T.; Schiavi, F.; Pawlu, C.; et al. Germline NF1 mutational spectra and loss-of-heterozygosity analyses in patients with pheochromocytoma and neurofibromatosis type 1. J. Clin. Endocrinol. Metab. 2007, 92, 2784-2792. [CrossRef]

5. Jochmanova, I.; Yang, C.; Zhuang, Z.; Pacak, K. Hypoxia-inducible factor signaling in pheochromocytoma: Turning the rudder in the right direction. J. Natl. Cancer Inst. 2013, 105, 1270-1283. [CrossRef]

6. Crona, J.; Taieb, D.; Pacak, K. New Perspectives on Pheochromocytoma and Paraganglioma: Toward a Molecular Classification. Endocr. Rev. 2017, 38, 489-515. [CrossRef]

7. Dahia, P.L. Pheochromocytoma and paraganglioma pathogenesis: Learning from genetic heterogeneity. Nat. Rev. Cancer 2014, 14, 108-119. [CrossRef]

8. Fishbein, L.; Leshchiner, I.; Walter, V.; Danilova, L.; Robertson, A.G.; Johnson, A.R.; Lichtenberg, T.M.; Murray, B.A.; Ghayee, H.K.; Else, T.; et al. Comprehensive Molecular Characterization of Pheochromocytoma and Paraganglioma. Cancer Cell 2017, 31, 181-193. [CrossRef]

9. Fishbein, L.; Khare, S.; Wubbenhorst, B.; DeSloover, D.; D'Andrea, K.; Merrill, S.; Cho, N.W.; Greenberg, R.A.; Else, T.; Montone, K.; et al. Whole-exome sequencing identifies somatic ATRX mutations in pheochromocytomas and paragangliomas. Nat. Commun. 2015, 6, 6140. [CrossRef]

10. Job, S.; Draskovic, I.; Burnichon, N.; Buffet, A.; Cros, J.; Lepine, C.; Venisse, A.; Robidel, E.; Verkarre, V.; Meatchi, T.; et al. Telomerase activation and ATRX mutations are independent risk factors for metastatic pheochromocytoma and paraganglioma. Clin. Cancer Res. 2018, 25, 760-770. [CrossRef]

11. Neumann, H.P.; Pawlu, C.; Peczkowska, M.; Bausch, B.; McWhinney, S.R.; Muresan, M.; Buchta, M.; Franke, G.; Klisch, J.; Bley, T.A.; et al. Distinct clinical features of paraganglioma syndromes associated with SDHB and SDHD gene mutations. JAMA 2004, 292, 943-951. [CrossRef] [PubMed] 
12. Amar, L.; Bertherat, J.; Baudin, E.; Ajzenberg, C.; Bressac-de Paillerets, B.; Chabre, O.; Chamontin, B.; Delemer, B.; Giraud, S.; Murat, A.; et al. Genetic testing in pheochromocytoma or functional paraganglioma. J. Clin. Oncol. 2005, 23, 8812-8818. [CrossRef] [PubMed]

13. Turkova, H.; Prodanov, T.; Maly, M.; Martucci, V.; Adams, K.; Widimsky, J., Jr.; Chen, C.C.; Ling, A.; Kebebew, E.; Stratakis, C.A.; et al. Characteristics and Outcomes of Metastatic Sdhb and Sporadic Pheochromocytoma/Paraganglioma: An National Institutes of Health Study. Endocr. Pract. 2016, 22, 302-314. [CrossRef]

14. Gimenez-Roqueplo, A.P.; Favier, J.; Rustin, P.; Rieubland, C.; Crespin, M.; Nau, V.; Khau Van Kien, P.; Corvol, P.; Plouin, P.F.; Jeunemaitre, X.; et al. Mutations in the SDHB gene are associated with extra-adrenal and/or malignant phaeochromocytomas. Cancer Res. 2003, 63, 5615-5621. [PubMed]

15. Nomura, K.; Kimura, H.; Shimizu, S.; Kodama, H.; Okamoto, T.; Obara, T.; Takano, K. Survival of patients with metastatic malignant pheochromocytoma and efficacy of combined cyclophosphamide, vincristine, and dacarbazine chemotherapy. J. Clin. Endocrinol. Metab. 2009, 94, 2850-2856. [CrossRef] [PubMed]

16. Huang, H.; Abraham, J.; Hung, E.; Averbuch, S.; Merino, M.; Steinberg, S.M.; Pacak, K.; Fojo, T. Treatment of malignant pheochromocytoma/paraganglioma with cyclophosphamide, vincristine, and dacarbazine: Recommendation from a 22-year follow-up of 18 patients. Cancer 2008, 113, 2020-2028. [CrossRef] [PubMed]

17. Niemeijer, N.D.; Alblas, G.; van Hulsteijn, L.T.; Dekkers, O.M.; Corssmit, E.P. Chemotherapy with cyclophosphamide, vincristine and dacarbazine for malignant paraganglioma and pheochromocytoma: Systematic review and meta-analysis. Clin. Endocrinol. 2014, 81, 642-651. [CrossRef]

18. Hadoux, J.; Favier, J.; Scoazec, J.Y.; Leboulleux, S.; Al Ghuzlan, A.; Caramella, C.; Deandreis, D.; Borget, I.; Loriot, C.; Chougnet, C.; et al. SDHB mutations are associated with response to temozolomide in patients with metastatic pheochromocytoma or paraganglioma. Int. J. Cancer 2014, 135, 2711-2720. [CrossRef]

19. Sulkowski, P.L.; Sundaram, R.K.; Oeck, S.; Corso, C.D.; Liu, Y.; Noorbakhsh, S.; Niger, M.; Boeke, M.; Ueno, D.; Kalathil, A.N.; et al. Krebs-cycle-deficient hereditary cancer syndromes are defined by defects in homologous-recombination DNA repair. Nat. Genet. 2018, 50, 1086-1092. [CrossRef]

20. Pang, Y.; Lu, Y.; Caisova, V.; Liu, Y.; Bullova, P.; Huynh, T.T.; Zhou, Y.; Yu, D.; Frysak, Z.; Hartmann, I.; et al. Targeting NAD+/PARP DNA repair pathway as a novel therapeutic approach to SDHB-mutated cluster I pheochromocytoma and paraganglioma. Clin. Cancer Res. 2018, 24, 3423-3432. [CrossRef]

21. Owens, K.M.; Aykin-Burns, N.; Dayal, D.; Coleman, M.C.; Domann, F.E.; Spitz, D.R. Genomic instability induced by mutant succinate dehydrogenase subunit $\mathrm{D}$ (SDHD) is mediated by $\mathrm{O}_{2}^{-\bullet}$ and $\mathrm{H}_{2} \mathrm{O}_{2}$. Free Radic. Biol. Med. 2012, 52, 160-166. [CrossRef] [PubMed]

22. Sporn, M.B.; Liby, K.T. NRF2 and cancer: The good, the bad and the importance of context. Nat. Rev. Cancer 2012, 12, 564-571. [CrossRef] [PubMed]

23. Ohta, T.; Iijima, K.; Miyamoto, M.; Nakahara, I.; Tanaka, H.; Ohtsuji, M.; Suzuki, T.; Kobayashi, A.; Yokota, J.; Sakiyama, T.; et al. Loss of Keap1 function activates Nrf2 and provides advantages for lung cancer cell growth. Cancer Res. 2008, 68, 1303-1309. [CrossRef] [PubMed]

24. Ren, D.; Villeneuve, N.F.; Jiang, T.; Wu, T.; Lau, A.; Toppin, H.A.; Zhang, D.D. Brusatol enhances the efficacy of chemotherapy by inhibiting the Nrf2-mediated defense mechanism. Proc. Natl. Acad. Sci. USA 2011, 108, 1433-1438. [CrossRef] [PubMed]

25. Guzy, R.D.; Sharma, B.; Bell, E.; Chandel, N.S.; Schumacker, P.T. Loss of the SdhB, but Not the SdhA, subunit of complex II triggers reactive oxygen species-dependent hypoxia-inducible factor activation and tumorigenesis. Mol. Cell. Biol. 2008, 28, 718-731. [CrossRef] [PubMed]

26. Yankovskaya, V.; Horsefield, R.; Tornroth, S.; Luna-Chavez, C.; Miyoshi, H.; Leger, C.; Byrne, B.; Cecchini, G.; Iwata, S. Architecture of succinate dehydrogenase and reactive oxygen species generation. Science 2003, 299, 700-704. [CrossRef]

27. Quinlan, C.L.; Orr, A.L.; Perevoshchikova, I.V.; Treberg, J.R.; Ackrell, B.A.; Brand, M.D. Mitochondrial complex II can generate reactive oxygen species at high rates in both the forward and reverse reactions. J. Biol. Chem. 2012, 287, 27255-27264. [CrossRef]

28. Bezawork-Geleta, A.; Wen, H.; Dong, L.; Yan, B.; Vider, J.; Boukalova, S.; Krobova, L.; Vanova, K.; Zobalova, R.; Sobol, M.; et al. Alternative assembly of respiratory complex II connects energy stress to metabolic checkpoints. Nat. Commun. 2018, 9, 2221. [CrossRef]

29. Ricketts, C.; Woodward, E.R.; Killick, P.; Morris, M.R.; Astuti, D.; Latif, F.; Maher, E.R. Germline SDHB mutations and familial renal cell carcinoma. J. Natl. Cancer Inst. 2008, 100, 1260-1262. [CrossRef] 
30. Neumann, H.P.; Bausch, B.; McWhinney, S.R.; Bender, B.U.; Gimm, O.; Franke, G.; Schipper, J.; Klisch, J.; Altehoefer, C.; Zerres, K.; et al. Germ-line mutations in nonsyndromic pheochromocytoma. N. Engl. J. Med. 2002, 346, 1459-1466. [CrossRef]

31. Panieri, E.; Santoro, M.M. ROS homeostasis and metabolism: A dangerous liason in cancer cells. Cell Death Dis. 2016, 7, e2253. [CrossRef]

32. Lushchak, V.I. Free radicals, reactive oxygen species, oxidative stress and its classification. Chem. Biol. Interact. 2014, 224, 164-175. [CrossRef]

33. Itoh, K.; Chiba, T.; Takahashi, S.; Ishii, T.; Igarashi, K.; Katoh, Y.; Oyake, T.; Hayashi, N.; Satoh, K.; Hatayama, I.; et al. An Nrf2/small Maf heterodimer mediates the induction of phase II detoxifying enzyme genes through antioxidant response elements. Biochem. Biophys. Res. Commun. 1997, 236, 313-322. [CrossRef]

34. Jaramillo, M.C.; Zhang, D.D. The emerging role of the Nrf2-Keap1 signaling pathway in cancer. Genes Dev. 2013, 27, 2179-2191. [CrossRef]

35. Itoh, K.; Wakabayashi, N.; Katoh, Y.; Ishii, T.; Igarashi, K.; Engel, J.D.; Yamamoto, M. Keap1 represses nuclear activation of antioxidant responsive elements by Nrf2 through binding to the amino-terminal Neh2 domain. Genes Dev. 1999, 13, 76-86. [CrossRef] [PubMed]

36. Olayanju, A.; Copple, I.M.; Bryan, H.K.; Edge, G.T.; Sison, R.L.; Wong, M.W.; Lai, Z.Q.; Lin, Z.X.; Dunn, K.; Sanderson, C.M.; et al. Brusatol provokes a rapid and transient inhibition of Nrf2 signaling and sensitizes mammalian cells to chemical toxicity-implications for therapeutic targeting of Nrf2. Free Radic. Biol. Med. 2015, 78, 202-212. [CrossRef] [PubMed]

37. Cai, S.J.; Liu, Y.; Han, S.; Yang, C. Brusatol, an NRF2 inhibitor for future cancer therapeutic. Cell Biosci. 2019, 9, 45. [CrossRef] [PubMed]

38. Liu, Y.; Lu, Y.; Celiku, O.; Li, A.; Wu, Q.; Zhou, Y.; Yang, C. Targeting IDH1-Mutated Malignancies with NRF2 Blockade. J. Natl. Cancer Inst. 2019, 111, 1033-1041. [CrossRef] [PubMed]

39. Tang, X.; Fu, X.; Liu, Y.; Yu, D.; Cai, S.J.; Yang, C. Blockade of Glutathione Metabolism in IDH1-Mutated Glioma. Mol. Cancer Ther. 2019, 19, 221-230. [CrossRef]

40. Sun, X.; Wang, Q.; Wang, Y.; Du, L.; Xu, C.; Liu, Q. Brusatol Enhances the Radiosensitivity of A549 Cells by Promoting ROS Production and Enhancing DNA Damage. Int. J. Mol. Sci. 2016, 17, 997. [CrossRef] [PubMed]

41. Lu, Z.; Lai, Z.Q.; Leung, A.W.N.; Leung, P.S.; Li, Z.S.; Lin, Z.X. Exploring brusatol as a new anti-pancreatic cancer adjuvant: Biological evaluation and mechanistic studies. Oncotarget 2017, 8, 84974-84985. [CrossRef] [PubMed]

42. Ghayee, H.K.; Bhagwandin, V.J.; Stastny, V.; Click, A.; Ding, L.H.; Mizrachi, D.; Zou, Y.S.; Chari, R.; Lam, W.L.; Bachoo, R.M.; et al. Progenitor cell line (hPheo1) derived from a human pheochromocytoma tumor. PLoS ONE 2013, 8, e65624. [CrossRef] [PubMed]

43. Ito, H.; Fujita, K.; Tagawa, K.; Chen, X.; Homma, H.; Sasabe, T.; Shimizu, J.; Shimizu, S.; Tamura, T.; Muramatsu, S.; et al. HMGB1 facilitates repair of mitochondrial DNA damage and extends the lifespan of mutant ataxin-1 knock-in mice. EMBO Mol. Med. 2015, 7, 78-101. [CrossRef]

44. Lu, Y.; Liu, Y.; Yang, C. Evaluating In Vitro DNA Damage Using Comet Assay. J. Vis. Exp. 2017, 128 , e56450. [CrossRef] [PubMed]

45. Yang, C.; Sun, M.G.; Matro, J.; Huynh, T.T.; Rahimpour, S.; Prchal, J.T.; Lechan, R.; Lonser, R.; Pacak, K.; Zhuang, Z. Novel HIF2A mutations disrupt oxygen sensing, leading to polycythemia, paragangliomas, and somatostatinomas. Blood 2013, 121, 2563-2566. [CrossRef]

46. Yang, C.; Wang, H.; Zhu, D.; Hong, C.S.; Dmitriev, P.; Zhang, C.; Li, Y.; Ikejiri, B.; Brady, R.O.; Zhuang, Z. Mutant glucocerebrosidase in Gaucher disease recruits Hsp27 to the Hsp90 chaperone complex for proteasomal degradation. Proc. Natl. Acad. Sci. USA 2015, 112, 1137-1142. [CrossRef]

47. Pang, Y.; Yang, C.; Schovanek, J.; Wang, H.; Bullova, P.; Caisova, V.; Gupta, G.; Wolf, K.I.; Semenza, G.L.; Zhuang, Z.; et al. Anthracyclines suppress pheochromocytoma cell characteristics, including metastasis, through inhibition of the hypoxia signaling pathway. Oncotarget 2017, 8, 22313-22324. [CrossRef]

(C) 2020 by the authors. Licensee MDPI, Basel, Switzerland. This article is an open access article distributed under the terms and conditions of the Creative Commons Attribution (CC BY) license (http://creativecommons.org/licenses/by/4.0/). 